\title{
Geometry of Work Fluctuations versus Efficiency in Microscopic Thermal Machines
}

\author{
Harry J. D. Miller $\oplus^{1}$ and Mohammad Mehboudi $\oplus^{2,3}$ \\ ${ }^{1}$ Department of Physics and Astronomy, The University of Manchester, Manchester M13 9PL, United Kingdom \\ ${ }^{2}$ Max-Planck-Institut für Quantenoptik, D-85748 Garching, Germany \\ ${ }^{3}$ ICFO-Institut de Ciencies Fotoniques, The Barcelona Institute of Science and Technology, \\ 08860 Castelldefels (Barcelona), Spain
}

(Received 21 September 2020; accepted 24 November 2020; published 22 December 2020)

\begin{abstract}
When engineering microscopic machines, increasing efficiency can often come at a price of reduced reliability due to the impact of stochastic fluctuations. Here we develop a general method for performing multiobjective optimization of efficiency and work fluctuations in thermal machines operating close to equilibrium in either the classical or quantum regime. Our method utilizes techniques from thermodynamic geometry, whereby we match optimal solutions to protocols parametrized by their thermodynamic length. We characterize the optimal protocols for continuous-variable Gaussian machines, which form a crucial class in the study of thermodynamics for microscopic systems.
\end{abstract}

DOI: 10.1103/PhysRevLett.125.260602

Designing optimal protocols for heat-to-work conversion below the nanoscale remains an ongoing challenge in the fields of stochastic and quantum thermodynamics [1-3]. For microscopic machines, efficiency and power alone are not the only figures of merit due to the additional influence of stochastic fluctuations. While a machine may extract work efficiently on average, it may be subject to large work fluctuations which hampers any reliability. Considerable effort is now being devoted to study this interplay between efficiency, power, and reliability in small scale systems [4-13].

Dissipation can be minimized for general far-fromequilibrium processes using methods from optimal control theory [14-19]. However finding solutions with such approaches is often limited to simple systems with few degrees of freedom, which makes it difficult to identify general design principles for efficient thermal machines beyond specific models. On the other hand, a general method for optimizing the efficiencies of machines operating close to equilibrium was recently proposed by Brandner and Saito [20]. This method, applicable to both classical and quantum periodic heat engines, relies on expressing the engine's entropy production in terms of a metric over the Riemann manifold of equilibrium states of the working system. One can maximize the efficiency for any given protocol by reparametrizing it in terms of the so-called thermodynamic length [21-31], which provides a measure of distance between configurations in the equilibrium manifold.

Published by the American Physical Society under the terms of the Creative Commons Attribution 4.0 International license. Further distribution of this work must maintain attribution to the author(s) and the published article's title, journal citation, and DOI. Open access publication funded by the Max Planck Society.
The benefit of this approach is its simplicity; optimization is achieved by a straightforward computation of the thermodynamic metric tensor which depends only on the equilibrium and relaxation properties of the machine [20].

While thermodynamic length provides a systematic way of determining efficient protocols, it remains to be seen how increasing efficiency impacts the work fluctuations. For systems connected to a single fixed-temperature reservoir, initial investigations have explored the simultaneous optimization of the average dissipated work required to drive a system from one state to another and the associated minimal work fluctuations [9,32]. As this is a multi-objective optimization problem, one must consider the boundary of allowed protocols where dissipation cannot be reduced any further without suffering an increase in fluctuations, or vice versa. This boundary, known as a Pareto front [33], only converges to a single point in regimes where the fluctuationdissipation relation holds true [34-36], in which case there exists a unique protocol with minimal average dissipated work and work variance. At present, Pareto optimization has not been analyzed in the context of periodic heat engines operating between different temperatures. In this situation at least two figures of merit are thermodynamic efficiency versus the resulting work fluctuations, whose optimal protocols are not expected to coincide for both classical and quantum systems. This is due to the fact that efficiency and work fluctuations are typically not monotonically related to each other, which can prohibit the existence of a unique optimal protocol.

In this Letter, we outline a general method for finding Pareto-optimal protocols interpolating between maximum efficiency and minimal work fluctuations for engines operating close to equilibrium. Remarkably, we show that such protocols can be found by constructing a new form of 
thermodynamic metric tensor and corresponding length. By parameterizing any given protocol in terms of this generalized thermodynamic length, one may identify regimes of optimal efficiency and reliability in a straightforward manner. We illustrate our approach for quantum heat engines operating along discrete step-equilibration cycles [37-40], though our results also apply to classical heat engines and open quantum systems undergoing Lindblad dynamics. As a core application, we derive analytic expressions for thermodynamic length in general continuous variable Gaussian quantum systems [41]. Such systems form a major platform for studying thermodynamic processes in the microscopic regime, and our approach can be used to optimize any Gaussian thermal machine by using the natural tools in the Gaussian formalism that operate on the steady-state covariance matrix. As an example, we determine Pareto fronts of optimal efficiency and reliability for a system of coupled harmonic oscillators driven by periodic changes in temperature, frequency, and coupling strength.

Let us begin by considering a quantum system weakly coupled to a thermal environment at inverse temperature $\beta=1 / k_{B} T$. The system is subject to external control via a set of $d$ mechanical parameters $\vec{\lambda}:=\left(\lambda^{1}, \lambda^{2}, \ldots \lambda^{d}\right)$ and we denote its Hamiltonian by $H(\vec{\lambda})$. In addition, we allow for external modulation of the environmental temperature. Collectively these sets of variables define a cycle via a closed curve $\gamma: t \mapsto \vec{\Lambda}_{t}$ in the parameter space containing the vectors

$$
\vec{\Lambda}:=\{\beta, \vec{\lambda}\} \in \mathbb{R}^{d+1},
$$

and we label $\Lambda^{0}=\beta$ and $\Lambda^{j}=\lambda^{j}$ for $j \geq 1$. The parameter space defines a manifold of equilibrium states, defined by $\pi(\vec{\Lambda}):=\exp [-\beta H(\vec{\lambda})] / \operatorname{Tr}\{\exp [-\beta H(\vec{\lambda})]\}$. Furthermore, we will introduce the following conjugate forces:

$$
X_{j}(\vec{\Lambda}):= \begin{cases}\beta^{-1} H(\vec{\lambda}), & \text { if } j=0, \\ \frac{\partial}{\partial \Lambda^{j}} H(\vec{\lambda}), & \text { if } j \geq 1 .\end{cases}
$$

During the cycle the inverse temperature undergoes a variation between a maximum and minimum, $\beta_{h} \leq \beta(t) \leq \beta_{c}$, which we will express in the form $\beta(t):=$ $\beta_{c}+\left(\beta_{h}-\beta_{c}\right) \delta \beta(t)$ with $\delta \beta(t) \in[0,1]$ a dimensionless periodic function. For convenience we take $t \in[0,1]$ to be a dimensionless parameter, and denote a discretized set of $N$ points along the curve evaluated at times $t_{n}=$ $(n-1) /(N-1)$ for $n \in[1, N]$. We have in mind thermodynamic cycles, where each step can be approximated by a fast quench in the mechanical parameters $\vec{\lambda}_{t_{n}} \rightarrow \vec{\lambda}_{t_{n+1}}$, followed by relaxation to a new temperature $\beta_{t_{n}} \rightarrow \beta_{t_{n+1}}$. This means that at the beginning of each $n$th step the system is in a thermal state $\pi\left(\vec{\Lambda}_{t_{n}}\right)$, which is left unchanged during the quench step while work is performed. The state after the quench then relaxes to a new equilibrium state $\pi\left(\vec{\Lambda}_{t_{n+1}}\right)$ with no work done during this step.
A central quantity of interest is the average irreversible entropy production along the cycle $\gamma$, which can be expressed as

$S_{i r r}:=\beta_{c} W+\left(\beta_{c}-\beta_{h}\right) Q_{\text {in }}=\sum_{n=1}^{N-1} S\left[\pi\left(\vec{\Lambda}_{t_{n}}\right) \| \pi\left(\vec{\Lambda}_{t_{n+1}}\right)\right]$,

where $S\left[\rho \| \rho^{\prime}\right]=\operatorname{Tr}[\rho \ln (\rho)]-\operatorname{Tr}\left[\rho \ln \left(\rho^{\prime}\right)\right] \geq 0$ the quantum relative entropy, and we identify the work done,

$$
W:=\sum_{n=1}^{N-1} \operatorname{Tr}\left\{\left[H\left(\vec{\lambda}_{t_{n+1}}\right)-H\left(\vec{\lambda}_{t_{n}}\right)\right] \pi\left(\vec{\Lambda}_{t_{n}}\right)\right\}
$$

and supplied heat

$$
Q_{\text {in }}:=\sum_{n=1}^{N-1} \delta \beta\left(t_{n+1}\right) \operatorname{Tr}\left\{H\left(\vec{\lambda}_{t_{n}}\right)\left[\pi\left(\vec{\Lambda}_{t_{n+1}}\right)-\pi\left(\vec{\Lambda}_{t_{n}}\right]\right\} .\right.
$$

The second equality in Eq. (3) follows from using the periodicity $\vec{\Lambda}_{t_{1}}=\vec{\Lambda}_{t_{N}}$ [42]. This formula motivates a definition of efficiency for processes with a positive work output, $W \leq 0$, given by the ratio $[51,52]$

$$
\eta:=-\frac{W}{Q_{\text {in }}} \leq \eta_{C}
$$

where $\eta_{C}=1-\beta_{h} / \beta_{c}$ denotes the Carnot efficiency. Here we see consistency with Carnot's theorem, which follows as a consequence of the second law $S_{i r r} \geq 0$.

In addition to efficiency, we will also be concerned with the amount of work fluctuations generated along the cycle. For quantum systems, stochastic work is determined from projective measurements of the system energy at the beginning and end of each unitary step [53]. By summing up the changes in energy across each step and computing the variance from the resulting work probability distribution, one can show $[32,40]$

$$
\begin{aligned}
\operatorname{Var}(W):= & \sum_{n=1}^{N-1} \operatorname{Tr}\left\{\left[H\left(\vec{\lambda}_{t_{n+1}}\right)-H\left(\vec{\lambda}_{t_{n}}\right)\right]^{2} \pi\left(\vec{\Lambda}_{t_{n}}\right)\right\} \\
& -\operatorname{Tr}\left\{\left[H\left(\vec{\lambda}_{t_{n+1}}\right)-H\left(\vec{\lambda}_{t_{n}}\right)\right] \pi\left(\vec{\Lambda}_{t_{n}}\right)\right\}^{2} .
\end{aligned}
$$

At this stage, we restrict our attention to cycles composed of a large number of steps $N^{2} \gg 1$, which defines a regime that is close to quasistatic [37]. In this case we may replace the summation with an integral over the continuous path $\gamma$ and obtain

$$
\operatorname{Var}(W) \simeq \frac{1}{N} \int_{\gamma} d t m_{j k}\left(\vec{\Lambda}_{t}\right) \frac{d \lambda^{j}}{d t} \frac{d \lambda^{k}}{d t},
$$

where summation is carried out over repeated indices, restricted to the mechanical variables only. Here $m_{j k}$ is a positive semidefinite and symmetric tensor

$$
m_{j k}(\vec{\Lambda}):=\frac{1}{2} \operatorname{Tr}\left[\pi(\vec{\Lambda})\left\{\delta X_{j}(\vec{\Lambda}), \delta X_{k}(\vec{\Lambda})\right\}_{+}\right], \quad \text { if } j, k \geq 1
$$


and we set $m_{j 0}=m_{0 j}=0 \forall j$ for later convenience. We also denote the shifted operators $\delta X_{j}(\vec{\Lambda})=X_{j}(\vec{\Lambda})-$ $\operatorname{Tr}\left[X_{j}(\vec{\Lambda}) \pi(\vec{\Lambda})\right]$ and $\{,\}_{+}$represents the anticommutator. Turning to the efficiency, we consider the fraction of the efficiency below the Carnot value, defined as $\delta \eta:=1-\left(\eta / \eta_{C}\right)$. Assuming a large number of steps and applying a Taylor expansion in $1 / N$ to Eq. (3) gives

$$
\delta \eta \simeq-\frac{1}{2 N \beta_{c} \mathcal{W}} \int_{\gamma} d t g_{j k}\left(\vec{\Lambda}_{t}\right) \frac{d \Lambda^{j}}{d t} \frac{d \Lambda^{k}}{d t},
$$

where we now have a different metric tensor:

$g_{j k}(\vec{\Lambda}):=\beta \int_{0}^{\beta} d x \operatorname{Tr}\left\{\pi(\vec{\Lambda}) \delta X_{j}(\vec{\lambda}) \mathcal{U}_{i x, \vec{\lambda}}\left[\delta X_{k}(\vec{\Lambda})\right]\right\}$,

and we have introduced the unitary channel $\mathcal{U}_{\nu, \vec{\lambda}}[]=$. $e^{i \nu H(\vec{\lambda})}[.] e^{-i \nu H(\vec{\lambda})}$. We have further defined the adiabatic work done [20]:

$$
\mathcal{W}:=\oint_{\gamma} \operatorname{Tr}\left[X_{j}(\vec{\Lambda}) \pi(\vec{\Lambda})\right] d \lambda^{j},
$$

which is a geometric quantity independent of the parametrization and assumed negative $\mathcal{W} \leq 0$ to ensure a useful work extraction cycle. We provide a proof of Eqs. (9) and (11) in Sec. B of the Supplemental Material [42]. The tensor (11) is proportional to the Kubo-Mori Fisher information metric [54,55], which is a quantum analog of the classical Fisher-Rao metric. At this stage we observe that the elements of this tensor typically differ from $m_{j k}$ due to possible noncommutativity between the conjugate forces, i.e., if $\left[X_{j}, X_{k}\right] \neq 0$. In Sec. A of Ref. [42] we highlight the different information-geometric interpretations of these two metrics.

To establish our optimization problem, let us introduce the dimensionless multiobjective function

$$
\mathcal{I}_{\epsilon}:=\epsilon \operatorname{Var}(\tilde{W})+(1-\epsilon) \delta \eta, \quad \epsilon \in[0,1]
$$

where for convenience we have defined the work fluctuations in units of the cold temperature, $\operatorname{Var}(\tilde{W})=$ $\beta_{c}^{2} \operatorname{Var}(W)$. The question we now address is how long should the system spend at each point along the protocol $\gamma$ in order to maximize efficiency while minimizing fluctuations? This amounts to finding the best choice of parametrization $\gamma: t \rightarrow \vec{\Lambda}_{t}^{\prime}=\vec{\Lambda}_{\phi_{t}^{\epsilon}}$ with function $\phi_{t}^{\epsilon} \in$ $[0,1]$ to be determined so as to minimize the scalarized objective (13). Optimal parametrizations $\mathcal{I}_{\epsilon}^{*} \leq \mathcal{I}_{\epsilon}$ lie along sections of the Pareto fronts [33]; these points form the boundary of protocols where it is not possible to increase efficiency (i.e., reduce $\delta \eta$ ) without increasing work fluctuations, or conversely, reduce fluctuations without reducing efficiency. By combining Eqs. (8) with (10) and applying the Cauchy-Schwarz inequality we arrive at a geometric expression for the minimized objective function:

$$
\mathcal{I}_{\epsilon}^{*}=\frac{\mathcal{L}_{\epsilon}^{2}}{N}, \quad \text { where } \mathcal{L}_{\epsilon}=\oint_{\gamma} \sqrt{M_{j k}(\vec{\Lambda}) d \Lambda^{j} d \Lambda^{k}},
$$

and we define

$$
M_{j k}^{\epsilon}(\vec{\Lambda}):=\epsilon \beta_{c}^{2} m_{j k}(\vec{\Lambda})+\frac{(1-\epsilon)}{2 \beta_{c}|\mathcal{W}|} g_{j k}(\vec{\Lambda}) .
$$

This follows from the fact that $M_{j k}^{\epsilon}(\vec{\Lambda})$ gives another metric tensor, since it is formed by a positive-weighted linear combination of two metric tensors for $\epsilon \in[0,1]$. The function $\mathcal{L}_{\epsilon}$ may be interpreted as a generalized form of thermodynamic length [27], whose dependence on $\epsilon$ encodes information about the Pareto optimal solution. In the classical regime where we may describe the thermal system by a probability distribution $p(\vec{\Lambda})$, we may determine the equivalent tensor from the Fisher-Rao metric $F_{j k}(\vec{\Lambda})=$ $\left\langle\partial_{\Lambda_{j}} \ln p(\vec{\Lambda}) \partial_{\Lambda_{k}} \ln p(\vec{\Lambda})\right\rangle$ (see Sec. A of Supplemental Material [42]). In this case, Eq. (15) takes the form

$$
M_{j k}^{\epsilon}(\vec{\Lambda})=\left[\epsilon\left(\frac{T}{T_{c}}\right)^{2} \mu_{j k}+\frac{(1-\epsilon)}{2 \beta_{c}|\mathcal{W}|}\right] F_{j k}(\vec{\Lambda}),
$$

with $\mu_{j 0}=\mu_{0 k}=0 \forall j, k$ and $\mu_{j k}=1 \forall j, k>0$.

Crucially, these Pareto optimal solutions are determined by parameterizing the protocol $\gamma$ in terms of the modified thermodynamic length via the speed function $t \rightarrow \phi_{t}^{\epsilon}$ [56], which is obtained from the implicit equation

$$
t=\frac{1}{\mathcal{L}_{\epsilon}} \int_{0}^{\phi_{t}^{\epsilon}} d s \sqrt{M_{j k}^{\epsilon}\left(\vec{\Lambda}_{s}\right) \frac{d \Lambda^{j}}{d s} \frac{d \Lambda^{k}}{d s}} .
$$

This means that for any given protocol, optimization is achieved by changing the speed at which the curve is traversed by choosing a new parametrization $\gamma: t \mapsto \vec{\Lambda}_{t}^{\prime}=\vec{\Lambda}_{\phi_{t}^{\epsilon}}$. As long as the solution satisfies $\left(\left|\dot{\phi}_{t}\right| / N\right)^{2} \ll 1$ at all times, the system remains sufficiently close to equilibrium and the optimal protocols may be realized. There are two limiting cases of the geometric bound. For $\epsilon=1$ we obtain a geometric lower bound on the achievable work fluctuations:

$$
\operatorname{Var}(W) \geq \frac{\left(k_{B} T_{c}\right)^{2}}{N} \mathcal{L}_{1}^{2} .
$$

For $\epsilon=0$, we obtain a maximum upper bound on efficiency $\eta \leq \eta_{C}\left(1-\mathcal{L}_{0}^{2} / N\right)$. An analogous efficiency bound was previously obtained in Ref. [20] for continuous Lindblad dynamics.

The above construction gives a general recipe for finding Pareto optimal protocols for arbitrary quantum or classical systems, valid in regimes where the number of steps between equilibrium states is large. A particular class of systems that are frequently used to describe many relevant 

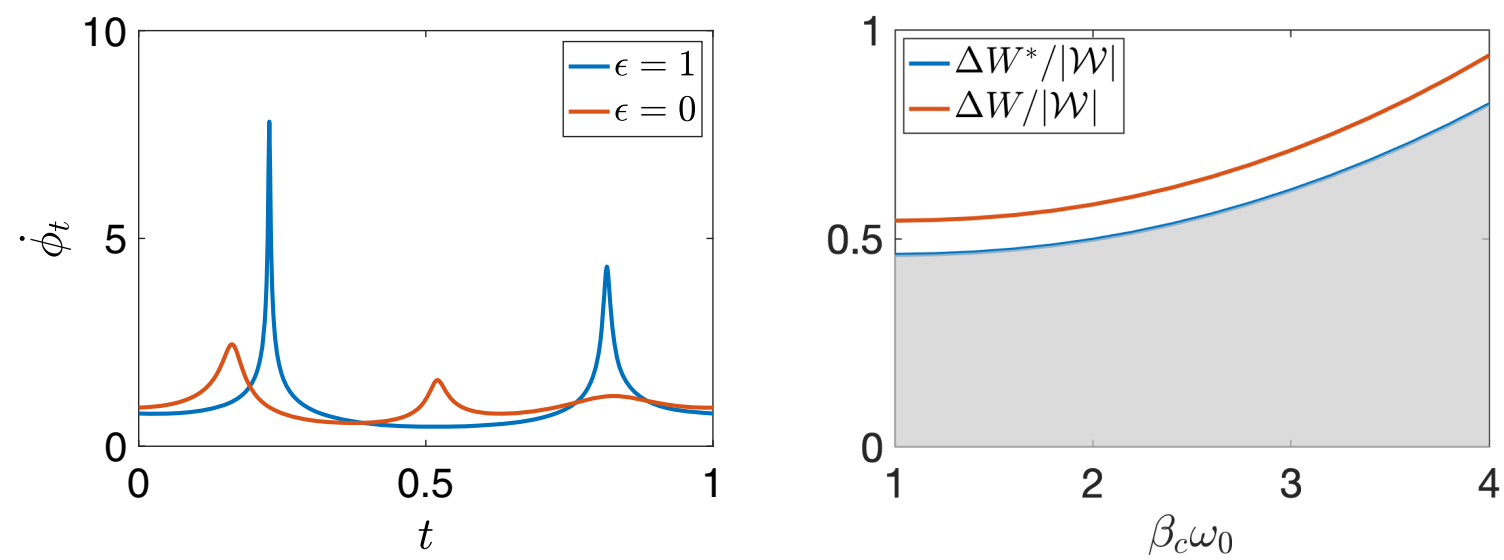

FIG. 1. Left—Derivative of the optimal speed $\phi_{t}^{\epsilon}$ (17), corresponding to work fluctuations optimization at $\epsilon=1$ (the blue curve) and efficiency optimization with $\epsilon=0$ (the red curve). The parameters are set to $\omega_{0}=1, T_{c}=0.25 \omega_{0}, T_{h}=T_{c}+\Delta T$ with $\Delta T=\omega_{0}$, $\kappa=0.4 \omega_{0}$. Right-Work fluctuations vs oscillator frequency $\omega_{0}$, for the linear protocol (red) and the optimal protocol (blue). We denote $\Delta W=\sqrt{\operatorname{Var}(W)}$ as the standard deviation and $\Delta W^{*}=k_{B} T_{c} \mathcal{L}_{1} / \sqrt{N}$ the optimal fluctuations given by Eq. (18). Both quantities are plotted relative to the adiabatic work extracted $|\mathcal{W}|$, which is independent of parametrization. The gray area is not accessible through any protocol. Here we set the parameters to $N=50, T_{c}=0.25, T_{h}=T_{c}+\Delta T$ with $\Delta T=4 T_{c}$, and $\kappa=0.4 T_{c}$.

physical systems in thermodynamics, such as ion trap heat engines [57], are composed of Gaussian quantum states [41,58]. The Hamiltonian of a $D$-mode Gaussian system is quadratic in quadrature operators, namely,

$$
H(\vec{\lambda})=\frac{1}{2} R^{T} \mathbb{G}_{\vec{\lambda}} R,
$$

with $R=\left(x_{1}, p_{1}, \ldots, x_{D}, p_{D}\right)^{T}$ being the quadrature vector. Here, the $D \times D$ dimensional symmetric matrix $\mathbb{G}_{\vec{\lambda}}$ contains all the quadratic couplings. For this general class we provide an analytic formula for the Pareto optimal solutions, which are given by Eq. (17) via computing the metric tensor (15). By defining $\mathbb{X}_{j}:=\partial_{\Lambda_{j}} \mathbb{G}_{\vec{\lambda}}$ if $j \geq 1$ and $\mathbb{X}_{0}:=\beta^{-1} \mathbb{G}_{\vec{\lambda}}$ we can express the metric (15) as follows (see Sec. D of the Supplemental Material [42]):

$$
\begin{aligned}
M_{j k}^{\epsilon}(\vec{\Lambda})= & a_{j k} \frac{\epsilon \beta_{c}^{2}}{4}\left\{\operatorname{tr}\left(\mathbb{X}_{j} \underline{\mathbb{X}}_{k}\right)+\operatorname{tr}\left(\mathbb{X}_{k} \underline{\mathbb{X}}_{j}\right)\right\} \\
& +\frac{(1-\epsilon) \beta}{4 \beta_{c}|\mathcal{W}|} \operatorname{tr}\left(\overline{\mathbb{X}}_{j} \underline{\mathbb{X}}_{k}\right),
\end{aligned}
$$

where $a_{j 0}=a_{0 j}=0 \forall j$ and $a_{j k}=1 \forall j, k>0$, and we define

$$
\begin{gathered}
\overline{\mathbb{X}}_{j}=\int_{0}^{\beta} d x\left[e^{i x \Omega \mathbb{G}_{\vec{\lambda}}}\right]^{T} \mathbb{X}_{j}\left[e^{i x \Omega \mathbb{G}_{\vec{\lambda}}}\right], \\
\underline{\mathbb{X}}_{j}=\left[\sigma(\vec{\Lambda})-\frac{1}{2} \Omega\right] \mathbb{X}_{j}\left[\sigma(\vec{\Lambda})+\frac{1}{2} \Omega\right],
\end{gathered}
$$

with $\Omega$ being the symplectic form with $\Omega_{n m}=i\left[R_{n}, R_{m}\right]$, and $\sigma(\vec{\Lambda})$ representing the steady state covariance matrix with elements $[\sigma(\vec{\Lambda})]_{n m}=\operatorname{Tr}\left[\pi(\vec{\Lambda})\left\{R_{n}, R_{m}\right\}_{+}\right] /$ $2-\operatorname{Tr}\left[\pi(\vec{\Lambda}) R_{n}\right] \operatorname{Tr}\left[\pi(\vec{\Lambda}) R_{m}\right]$. Furthermore, the adiabatic work is found using $\mathcal{W}=1 / 2 \oint_{\gamma} d \lambda^{j} \operatorname{tr}\left[\mathbb{X}_{j} \sigma(\vec{\Lambda})\right]$. Notice that the "tr" operation acts as a trace on the matrix space associated to the Gaussian covariance matrices, which should be distinguished from the trace "Tr" which acts on the Hilbert space for density operators.

We have now derived the general form for the thermodynamic metric tensor for Gaussian heat engines. So long as one can compute this metric tensor, the speed function $\phi_{t}^{\epsilon}$ can be approximately determined from Eq. (17) via pointwise inversion followed by numerical interpolation. We illustrate our method for the example of a pair of coupled harmonic oscillators with $R=\left[x_{1} p_{1} x_{2} p_{2}\right]^{T}$ and Hamiltonian coefficient matrix

$$
\mathbb{G}_{\vec{\lambda}}=\left[\begin{array}{cccc}
\omega^{2}+\kappa & 0 & -\kappa & 0 \\
0 & 1 & 0 & 0 \\
-\kappa & 0 & \omega^{2}+\kappa & 0 \\
0 & 0 & 0 & 1
\end{array}\right],
$$

were we chose equal frequencies $\omega_{1}=\omega_{2}=\omega$ and denote $\kappa$ the coupling strength between the oscillators. As for the driving protocol, we consider control over the bath temperature alongside the joint frequency and coupling, i.e., $\gamma: t \mapsto \overrightarrow{\Lambda_{t}}=\{\beta(t), \omega(t), \kappa(t)\}$. The matrices $\mathbb{X}_{\omega}=\partial_{\omega} \mathbb{G}_{\vec{\lambda}}$, $\mathbb{X}_{\kappa}=\partial_{\kappa} \mathbb{G}_{\vec{\lambda}}$, and $\mathbb{X}_{0}=\beta^{-1} \mathbb{G}_{\vec{\lambda}}$ are easily found from Eq. (23). By substituting in Eqs. (21) and (22) one finds the corresponding $\overline{\mathbb{X}}_{j}$ and $\underline{\mathbb{X}}_{j}$. Finally, by plugging these into Eq. (20) we find the metric [59]. We choose a harmonic protocol path $\beta(t)=\beta_{c}+\left(\beta_{h}-\beta_{c}\right) \sin ^{2}(\pi t)$, $\omega(t)=\omega_{0}\left[1+\sin ^{2}(\pi t+\pi / 4)\right], \quad$ and $\quad \kappa(t)=\kappa_{0}[1+$ $\left.\sin ^{2}(\pi t+\pi / 4)\right]$, with the parameters $\kappa_{0}, \omega_{0}, \beta_{c}$, and $\beta_{h}$ being fixed during the cycle. In Fig. 1 we compare the work 

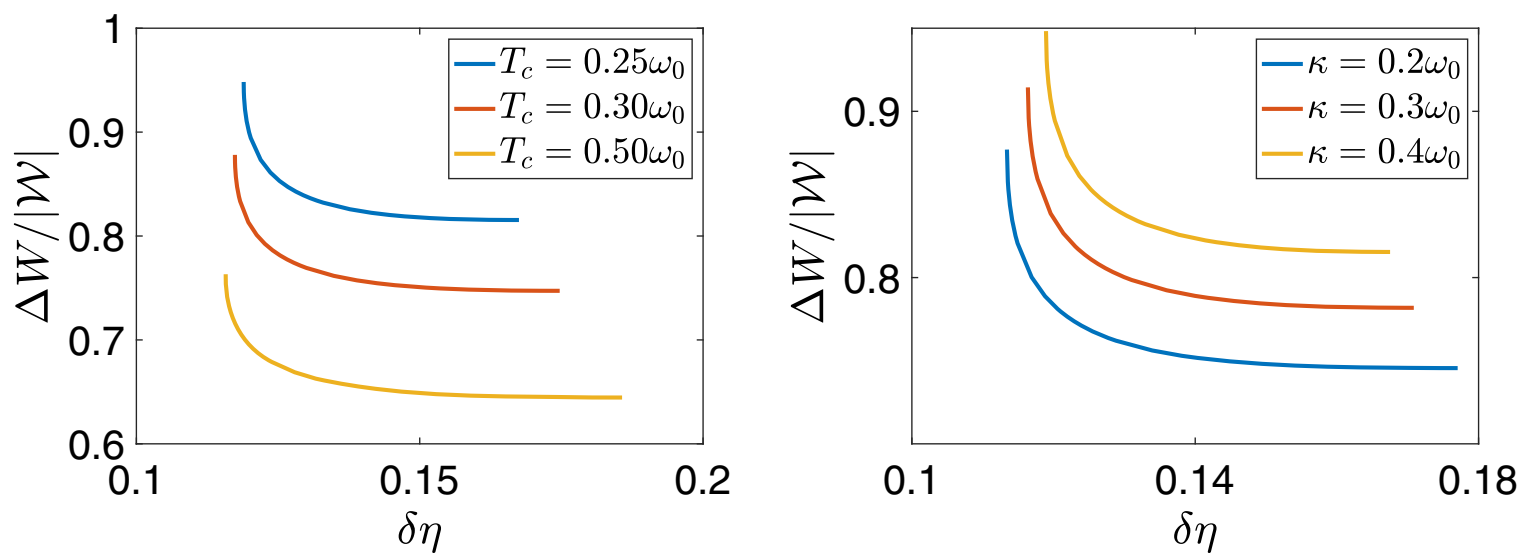

FIG. 2. Left-The relative work fluctuations vs efficiency Pareto fronts for different values of cold temperature $T_{c}$. Overall neither the relative work fluctuations nor the efficiency have a monotonic behavior with temperature. The parameters are set to $\omega_{0}=2, T_{h}=$ $T_{c}+\Delta T$ with $\Delta T=\omega_{0}, \kappa=0.4 \omega_{0}$. Right - Same as left, for different couplings between the two oscillators. Here we set $\omega_{0}=1$, $T_{c}=0.25 \omega_{0}, T_{h}=T_{c}+\Delta T$ with $\Delta T=\omega_{0}$ and $N=50$.

fluctuations $\Delta W=\sqrt{\operatorname{Var}(W)}$ for a linear parametrization as a function of the optimal amount $\Delta W^{*}=$ $k_{B} T_{c} \mathcal{L}_{1} / \sqrt{N}$ given by Eq. (18), both as a function of oscillator frequency $\omega_{0}$ and expressed in units of the adiabatic work. We also plot the rate of change in the speed function $\phi_{t}^{\epsilon}$ versus time for $\epsilon=1$, giving minimal fluctuations, compared with $\epsilon=0$ that gives maximum efficiency. One can clearly see that distinct protocols must be chosen in order to achieve either optimal efficiency or fluctuations. In Fig. 2 we plot the Pareto fronts for the model for different choices of cold temperature $T_{c}$ and coupling constant $\kappa$. The points on each curve give the corresponding values of $\delta \eta$ and $\Delta W$ for the optimal protocol $\vec{\Lambda}_{t}^{\prime}=\vec{\Lambda}_{\phi_{t}^{\varepsilon}}$ determined from Eq. (17) for every $\epsilon \in[0,1]$. These curves form the boundary of achievable fluctuations and efficiency for the chosen protocol $\gamma$, with points below the curves inaccessible. In this case the Pareto fronts are strictly convex, and hence the entire front is determined by the minima of the scalarized objective function (13) [60].

To summarize, we have constructed a general method for performing multiobjective optimization of efficiency and work fluctuations in microscopic heat engines operating close to equilibrium. This method relies on determining a thermodynamic metric tensor that encodes information about both the efficiency and fluctuations simultaneously. While we have here focused on the quantum regime, our method can be readily applied to classical-stochastic systems using the tensor (16), which we demonstrate in Sec. C of the Supplemental Material [42] for the optimization of a classical harmonic oscillator. This formalism opens an avenue to further applications and generalizations. For example, in Ref. [42] we show that an analogous metric providing Pareto optimal solutions for work fluctuations and efficiency can be derived for engine cycles described using Lindblad dynamics, and we derive the corresponding expressions for Gaussian Lindbladians. Future investigations could focus on extending our approach to strongly coupled quantum heat engines [62], or regimes far away from equilibrium $[19,63]$. The Gaussian metric tensors we have derived can also be used to study other aspects of thermodynamic geometry, such as computing scalar curvature [26] and geodesics along the manifold of thermal Gaussian states [28].

We thank Martí Perarnau-Llobet for useful comments on the manuscript. This work was financially supported by Spanish MINECO (QIBEQI FIS2016-80773-P, ConTrAct FIS2017-83709- R, and Severo Ochoa SEV-2015-0522), Fundacio Privada Cellex, and the Generalitat de Catalunya (CERCA Program and SGR1381). H. J. D. M. acknowledges support from the Royal Commission for the Exhibition of 1851.

[1] U. Seifert, Rep. Prog. Phys. 75, 126001 (2012).

[2] G. Benenti, G. Casati, K. Saito, and R. S. Whitney, Phys. Rep. 694, 1 (2017).

[3] R. Kosloff and A. Levy, Annu. Rev. Phys. Chem. 65, 365 (2014).

[4] K. Funo and M. Ueda, Phys. Rev. Lett. 115, 260601 (2015).

[5] A. C. Barato and U. Seifert, Phys. Rev. Lett. 114, 158101 (2015).

[6] A. C. Barato and U. Seifert, Phys. Rev. X 6, 041053 (2016).

[7] P. Pietzonka and U. Seifert, Phys. Rev. Lett. 120, 190602 (2018).

[8] V. Holubec and A. Ryabov, Phys. Rev. Lett. 121, 120601 (2018).

[9] A. P. Solon and J. M. Horowitz, Phys. Rev. Lett. 120, 180605 (2018).

[10] J. M. Horowitz and T. R. Gingrich, Nat. Phys. 16, 15 (2020).

[11] G. Guarnieri, G. T. Landi, S. R. Clark, and J. Goold, Phys. Rev. Research 1, 033021 (2019). 
[12] P. Abiuso and M. Perarnau-Llobet, Phys. Rev. Lett. 124, 110606 (2020).

[13] T. Denzler and E. Lutz, arXiv:2007.01034.

[14] E. Aurell, C. Mejía-Monasterio, and P. MuratoreGinanneschi, Phys. Rev. Lett. 106, 250601 (2011).

[15] T. Schmiedl and U. Seifert, Phys. Rev. Lett. 98, 108301 (2007).

[16] P. R. Zulkowski and M. R. DeWeese, Phys. Rev. E 89, 052140 (2014).

[17] V. Cavina, A. Mari, A. Carlini, and V. Giovannetti, Phys. Rev. A 98, 012139 (2018).

[18] M. V. S. Bonanca and S. Deffner, Phys. Rev. E 98, 042103 (2018).

[19] T. V. Vu and Y. Hasegawa, arXiv:2005.02871.

[20] K. Brandner and K. Saito, Phys. Rev. Lett. 124, 040602 (2020).

[21] F. Weinhold, J. Chem. Phys. 63, 2479 (1975).

[22] F. Weinhold, J. Chem. Phys. 63, 2488 (1975).

[23] G. Ruppeiner, Phys. Rev. A 20, 1608 (1979).

[24] P. Salamon and R. S. Berry, Phys. Rev. Lett. 51, 1127 (1983).

[25] F. Schlögl, Z. Phys. B 59, 449 (1985).

[26] G. Ruppeiner, Rev. Mod. Phys. 67, 605 (1995).

[27] G. E. Crooks, Phys. Rev. Lett. 99, 100602 (2007).

[28] D. A. Sivak and G. E. Crooks, Phys. Rev. Lett. 108, 190602 (2012).

[29] P. R. Zulkowski, D. A. Sivak, G. E. Crooks, and M. R. Deweese, Phys. Rev. E 86, 041148 (2012).

[30] M. Scandi and M. Perarnau-Llobet, Quantum 3, 197 (2019).

[31] S. Deffner and M. V. S. Bonanca, Europhys. Lett. 131, 20001 (2020).

[32] H. J. D. Miller, M. Scandi, J. Anders, and M. PerarnauLlobet, Phys. Rev. Lett. 123, 230603 (2019).

[33] K. Miettinen, Nonlinear Multiobjective Optimization (Springer Science \& Business Media, New York, 1999).

[34] C. Jarzynski, Phys. Rev. Lett. 78, 2690 (1997).

[35] T. Speck and U. Seifert, Phys. Rev. E 70, 066112 (2004).

[36] D. Mandal and C. Jarzynski, J. Stat. Mech. (2016) 063204.

[37] J. Nulton, P. Salamon, B. Andresen, Q. Anmin, J. Nulton, P. Salamon, B. Andresen, and Q. Anmin, J. Chem. Phys. 83, 334 (1985).

[38] J. Anders and V. Giovannetti, New J. Phys. 15, 033022 (2013).

[39] S. J. Large and D. A. Sivak, J. Stat. Mech. (2019) 083212.

[40] M. Scandi, H. J. D. Miller, J. Anders, and M. PerarnauLlobet, Phys. Rev. Research 2, 023377 (2020).

[41] C. Weedbrook, S. Pirandola, R. Garcia-Patron, N. J. Cerf, T. C. Ralph, J. H. Shapiro, and S. Lloyd, Rev. Mod. Phys. 84, 621 (2012).
[42] See Supplemental Material at http://link.aps.org/ supplemental/10.1103/PhysRevLett.125.260602 for derivations and further discussions of the results presented in the main text. Sections A and B include Refs. [43,44], Sec. E include Refs. [45-50].

[43] M. Tomamichel, M. Hayashi, and S. Member, IEEE Trans. Inf. Theory 59, 7693 (2013).

[44] H. Janyszek, Rep. Math. Phys. 24, 11 (1986).

[45] R. Alicki and K. Lendi, Quantum Dynamical Semigroups and Applications (Springer, New York, 2007).

[46] V. Cavina, A. Mari, and V. Giovannetti, Phys. Rev. Lett. 119, 050601 (2017).

[47] H. J. D. Miller, M. H. Mohammady, M. Perarnau-Llobet, and G. Guarnieri, arXiv:2006.07316v2.

[48] B. Leggio, A. Napoli, A. Messina, and H.-P. Breuer, Phys. Rev. A 88, 042111 (2013).

[49] J. M. Horowitz and J. M. R. Parrondo, New J. Phys. 15, 085028 (2013).

[50] G. Manzano, J. M. Horowitz, and J. M. R. Parrondo, Phys. Rev. E 92, 032129 (2015).

[51] K. Brandner and U. Seifert, Phys. Rev. E 93, 062134 (2016).

[52] K. Brandner, K. Saito, and U. Seifert, Phys. Rev. X 5, 031019 (2015).

[53] P. Talkner, E. Lutz, and P. Hänggi, Phys. Rev. E 75, 050102 (R) (2007).

[54] D. Petz, J. Math. Phys. (N.Y.) 35, 780 (1994).

[55] M. Hayashi, J. Phys. A 35, 7689 (2002).

[56] In this case one has $t \propto s\left(\phi_{t}^{\epsilon}\right)$, where $s\left(\phi_{t}^{\epsilon}\right)$ is the arclength for the interval $\left[0, \phi_{t}^{\epsilon}\right]$. This means the curve $\gamma: t \mapsto \vec{\Lambda}_{t}$ is traversed at constant velocity, leading to the equality condition for the Cauchy-Schwartz inequality.

[57] J. Roßnagel, S. T. Dawkins, K. N. Tolazzi, O. Abah, E. Lutz, F. Schmidt-Kaler, and K. Singer, Science 352, 325 (2016).

[58] M. Mehboudi and J. M. R. Parrondo, New J. Phys. 21, 083036 (2019).

[59] The metric has a long and cumbersome analytical expression that we do not present.

[60] For a convex Pareto front, the full set of minima of $\mathcal{I}_{\epsilon}^{*} \forall \epsilon \in$ $[0,1]$ characterizes the entire front. However, points situated along any nonconvex regions of the front cannot be determined from minimizing $\mathcal{I}_{\epsilon}$ alone. In such situations, one can additionally apply an adaptive weighted sum method [61] to determine any nonconvex sections of the front.

[61] I. Y. Kim and O. L. D. Weck, Struct. Multidisc. Optim. 29, 149 (2005).

[62] D. Newman, F. Mintert, and A. Nazir, Phys. Rev. E 95, 032139 (2017).

[63] S. Deffner and E. Lutz, Phys. Rev. E 87, 022143 (2013). 